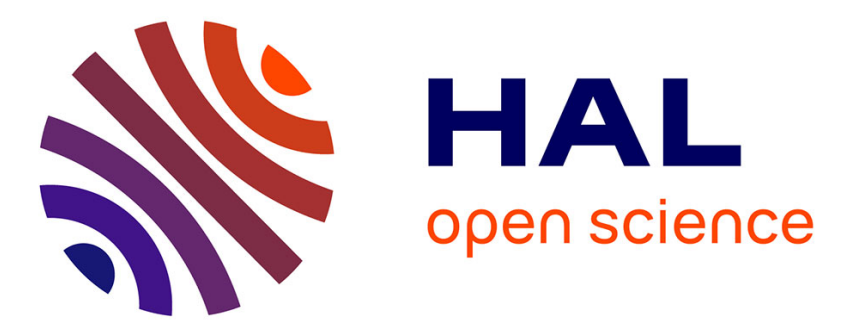

\title{
A frequency modulated wireless interrogation system exploiting narrowband acoustic resonator for remote physical quantity measurement
}

C. Droit, G. Martin, S. Ballandras, J.-M. Friedt

\section{- To cite this version:}

C. Droit, G. Martin, S. Ballandras, J.-M. Friedt. A frequency modulated wireless interrogation system exploiting narrowband acoustic resonator for remote physical quantity measurement. Review of Scientific Instruments, 2010, 81 (5), pp.056103. 10.1063/1.3402286 . hal-00565266

\section{HAL Id: hal-00565266 \\ https://hal.science/hal-00565266}

Submitted on 9 Apr 2021

HAL is a multi-disciplinary open access archive for the deposit and dissemination of scientific research documents, whether they are published or not. The documents may come from teaching and research institutions in France or abroad, or from public or private research centers.
L'archive ouverte pluridisciplinaire HAL, est destinée au dépôt et à la diffusion de documents scientifiques de niveau recherche, publiés ou non, émanant des établissements d'enseignement et de recherche français ou étrangers, des laboratoires publics ou privés.

\section{(c)(1)}

Distributed under a Creative Commons Attribution| 4.0 International License 


\title{
Note: A frequency modulated wireless interrogation system exploiting narrowband acoustic resonator for remote physical quantity measurement
}

\author{
C. Droit, ${ }^{1}$ G. Martin, ${ }^{1}$ S. Ballandras, ${ }^{1}$ and J.-M. Friedt ${ }^{2, a)}$ \\ ${ }^{1}$ Time and Frequency Department, FEMTO-ST, 32 Avenue de l'Observatoire, Besançon 25044, France \\ ${ }^{2}$ SENSeOR, 32 Avenue de l'Observatoire, Besançon 25044, France
}

(Received 30 January 2010; accepted 29 March 2010; published online 18 May 2010)

\begin{abstract}
We demonstrate the wireless conversion of frequency modulation to amplitude modulation by radio frequency resonators as means of accurately determining the resonance frequency of passive acoustoelectronic sensors. The emitted frequency modulated radio frequency pulses are generated by a pulsed radar for probing a surface acoustic wave based sensor. The sharp sign transition of the amplitude modulated received signal provides a signal on which a feedback loop is locked to monitor the resonance signal. The strategy is demonstrated using a full software implementation on a generic hardware, resulting in $2 \mathrm{~Hz}$ resolution at $1 \mathrm{~s}$ integration time limited by the proportional feedback loop. (C) 2010 American Institute of Physics. [doi:10.1063/1.3402286]
\end{abstract}

We have demonstrated the working principles of a frequency sweep wireless network-analyzerlike strategy for identifying the resonance frequency of acoustic resonators used as sensors. ${ }^{1}$ The full software implementation of this strategy allows for innovative strategies such as frequency hopping or adaptative frequency interrogation schemes as monitored by a microcontroller defining the frequency generated by a radio frequency (rf) direct digital synthesizer (DDS). Beyond identifying the resonance frequency of an acoustic sensor through the maximum returned power, we here demonstrate the use of another functionality of the DDS, namely the ability to generate a frequency modulated (FM) signal by sweeping an internal random access memory (RAM) filled with user defined values for selecting the generated frequency. We demonstrate the accuracy gain in resonance frequency measurement achieved by exploiting a signed phase signal for keeping a feedback loop close to a null-returned signal through the software implementation on the previously described hardware.

In a previous demonstration of the wireless interrogation of passive acoustic resonators, ${ }^{1}$ we presented a strategy in which a fixed frequency comb is sequentially emitted, the returned energy sampled, and the resulting transfer function is fitted to extract the resonance frequency. We will refer to this technique as the "fixed comb" strategy.

A resonator acts, through its transfer function, as a frequency-to-amplitude modulation converter. ${ }^{2}$ Hence, by exciting a narrowband resonator away from its resonance frequency with a signal FM at rate of $\omega_{m}$ on one of the resonance slopes, the returned signal is an amplitude modulated at $\omega_{m}$. At resonance frequency, where the first order coefficient for a polynomial development of the transfer function becomes null and the second order coefficient becomes dominant, the frequency modulation at $\omega_{m}$ becomes an amplitude modulation at $2 \omega_{m}$, with a contribution at $\omega_{m}$ close to zero. Above the resonance frequency, the frequency modulation at $\omega_{m}$ again becomes an amplitude modulation at

\footnotetext{
${ }^{\text {a)} E l e c t r o n i c ~ m a i l: ~ j m f r i e d t @ f e m t o-s t . f r . ~}$
}

$\omega_{m}$, but this time with a $90^{\circ}$ phase shift of the amplitude modulation with respect to the frequency modulation signal compared to the previous case (Fig. 1). ${ }^{3}$

The purpose of this experiment is a practical application for improving the accuracy of the remote measurement of wireless passive rf sensors. As a reminder of the hardware we use as embedded measurement setup, a central ARM7based microcontroller sequences all tasks for the wireless interrogation of passive acoustic sensors: A DDS is configured to generate a given frequency in the $34 \pm 0.85 \mathrm{MHz}$ range, which is mixed with a fixed frequency-Phase Locked Loop (PLL) multiplied-400 MHz oscillator. The resulting bandpass filtered signal at $434 \pm 0.85 \mathrm{MHz}$ is amplified and emitted through a duplexer. Switching under microcontroller control to the receiving stage once the resonator is loaded, the received signal is bandpass filtered, amplified, and fed to a wideband rf-power detector for digitization following an offset removal step and low-frequency amplification. Hence, for each emitted frequency, a single magnitude response is recorded, representative of the amount of energy stored in the resonator, i.e., how close the emitted frequency is to the resonance frequency of the transducer.

We implemented this strategy using the hardware described above, ${ }^{1}$ as a full software implementation:

- the internal RAM of the DDS is filled with values describing the sine-shaped evolution of the emitted frequency, i.e., a FM emission. The frequency modulation deviation-also known as the modulation index $B$-is set by programming the RAM with values spanning the wanted deviation. The speed at which this RAM is swept, defined by a programmable internal counter, defines the modulation carrier $\omega_{m}$.

- while the FM signal is continuously emitted, the switches connecting the antenna alternately to the emission and reception circuits is controlled by the same microcontroller used to program the DDS.

- the power detector is periodically sampled as the switches are programmed since the FM signal has been converted to an amplitude modulated signal.

The received signal is then bandpass filtered at $\omega_{m}$, with 


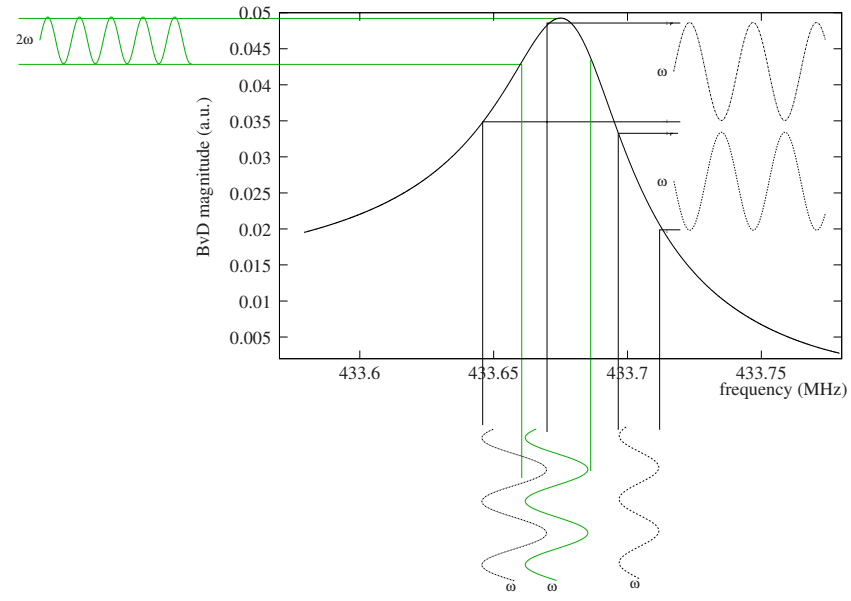

FIG. 1. (Color online) Frequency to amplitude modulation through the transfer function of the resonator: At resonance, the contribution at modulation pulsation $\omega_{m}$ vanishes and the sole contribution to the returned signal is at multiples of $2 \omega_{m}$. From left to right: When the slope of the transfer function is rising, the emitted FM signal is recovered as an amplitude modulated signal. At resonance frequency, the contribution at $\omega_{m}$ vanishes. When the slope of the transfer function is negative, the emitted signal at modulation frequency $\omega_{m}$ is received as an amplitude modulated signal at $\omega_{m}$ but with phase inversion.

removal of the dc component. We observe however that the received signal is biased by a value function of the received power: At resonance frequency, the $\omega_{m}$ component is removed (since it is converted to a solely $2 \omega_{m}$ component) but a background offset induces additional signal processing: The average value is subtracted in order to keep the resulting signal centered on the midscale of the analog-to-digital converter scale.

Furthermore, the modulation frequency carrier and excursion must be set according to some quantitative analysis. Let us consider an output signal $y(t)$ evolving with the time $t$ as

$$
y(t)=\cos \left[\omega_{c} \times t+B \times \sin \left(\omega_{m} \times t\right)\right],
$$

where $B$ is the modulation excursion, $\omega_{m}$ is the modulation frequency, and $\omega_{c}$ is the carrier frequency. The excursion is the easiest parameter to identify: It must be wide enough for the signal to significantly sweep the resonance (i.e., at least a few $\mathrm{kHz}$ ) without being larger than the width at half height of the resonance peak, i.e., less than $f_{0} / Q$ with $f_{0} \simeq 434 \mathrm{MHz}$ the resonance frequency of the sensor and $Q \simeq 10000$ its quality factor. The modulation frequency must be set considering that each response sampling must be performed assuming a quasistatic emitted signal loading the resonator. Considering the resonator parameters we provided above, and since the decay time of a resonator is $Q / \pi$ periods, each sampling step requires loading the resonator during $3 \tau=Q /\left(\pi \times f_{0}\right)=22 \mu \mathrm{s}$. The same delay is needed during the signal reception step. In order for the resonator to observe a quasistatic signal, we use a modulation carrier $(1 / 10) \times(1 / 2 \tau) \simeq 4.5 \mathrm{kHz}$. The received signal is sampled using a timer-defined periodic analog-to-digital conversion. The maximum allowed sampling speed, due to the other computational requirements, is $16 \mathrm{kHz}$ : The returned signal is recorded at the rate ranging from 4 to 5 points per period. We sample 10 periods for an accurate estimate of the returned signal amplitude. Hence, each frequency sampling-

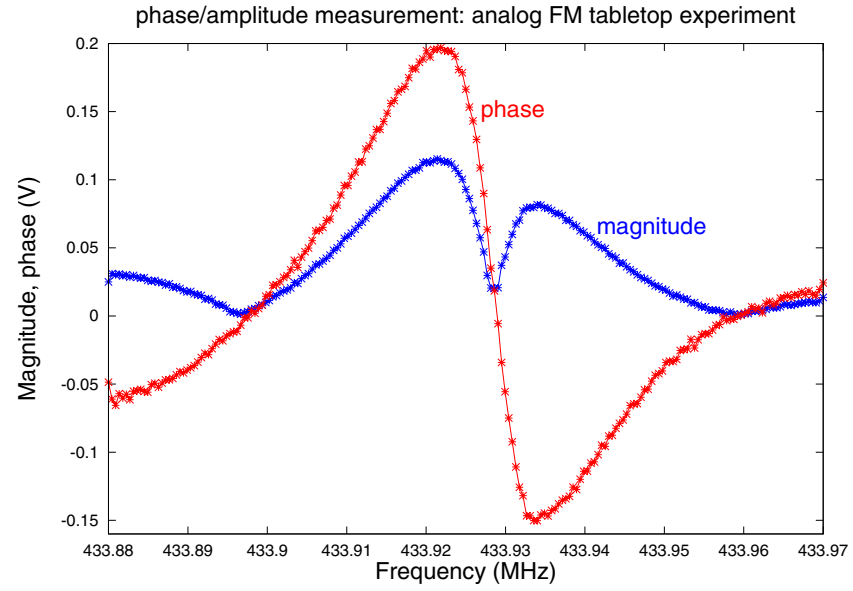

FIG. 2. (Color online) Open loop characterization-obtained using the tabletop setup (Ref. 6) — of the magnitude of the amplitude modulated signal at $\omega_{m}=10 \mathrm{kHz}$, and signed magnitude (phase). The signed amplitude (phase) is equal to the magnitude signal with the additional information of the received amplitude modulated signal position with respect to the emitted frequency modulation signal: the sign is +1 below the resonance frequency, and -1 above. The feedback control aims at keeping the phase information close to 0 .

and hence resonance frequency feedback control loop update-requires about $2.2 \mathrm{~ms}$.

The frequency demodulation would be expected to be restricted to a magnitude information since the timer defining the dates at which the received signal is sampled is not synchronized with the timer of the DDS defining how its internal RAM is swept. However, since the same microcontroller triggers the start of the RAM sweep, we can identify which sampled signal has been recorded as what instantaneous frequency was emitted. Hence, we can extract a phase information, and generate a signed feedback signal (Fig. 2).

The notch in the magnitude signal is replaced by a signed information which evolves linearly close to the resonance. While a notch is difficult to track since it requires, for best accuracy, a fine frequency tracking of the magnitude minimum, the linear signed magnitude signal is usable for a feedback.

Hence, we can select which samples are used for detecting the amplitude at maximum frequency modulation deviation, and extract the signed feedback signal for feeding a proportional controller.

We successfully implemented a full digital electronics version of this strategy, with the reference modulation signal implicit within the sweep rate of the look-up-table of the frequency synthesizer. The digital finite impulse response bandpass filter aims at removing the dc component of the received signal and keep only the signal at $\omega_{m}$ : The average received signal provides a rough estimate of the resonance frequency during an initial sweep, consistent with the measurement obtained using the fixed frequency comb strategy. Once the received average power is maximum, the control on the filtered signal keeps the emitted signal centered on the resonance frequency with a standard deviation of $25 \mathrm{~Hz}$, limited by the stability of the resonator. We have demonstrated sub-10 Hz stability with a resonator attached to a heat sink (Fig. 3, right). Assuming for instance the same kind of differential sensors as described in Ref. 1, with a measurement 


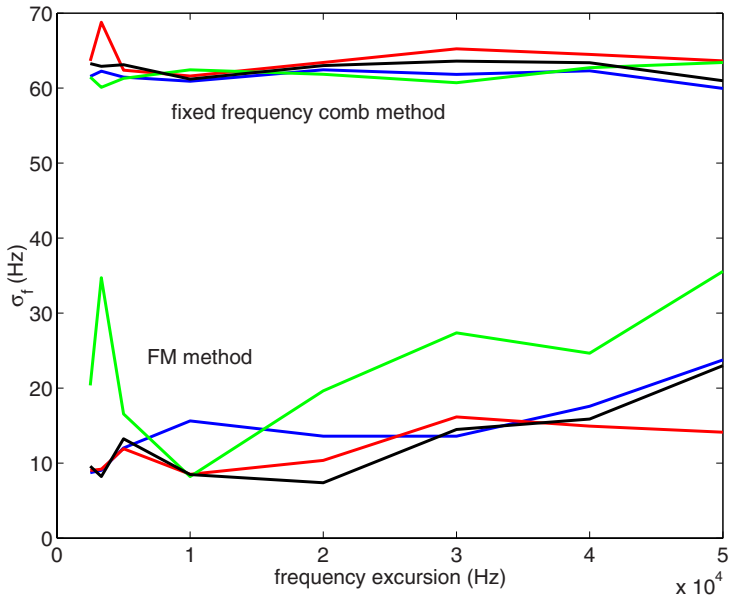

(a)

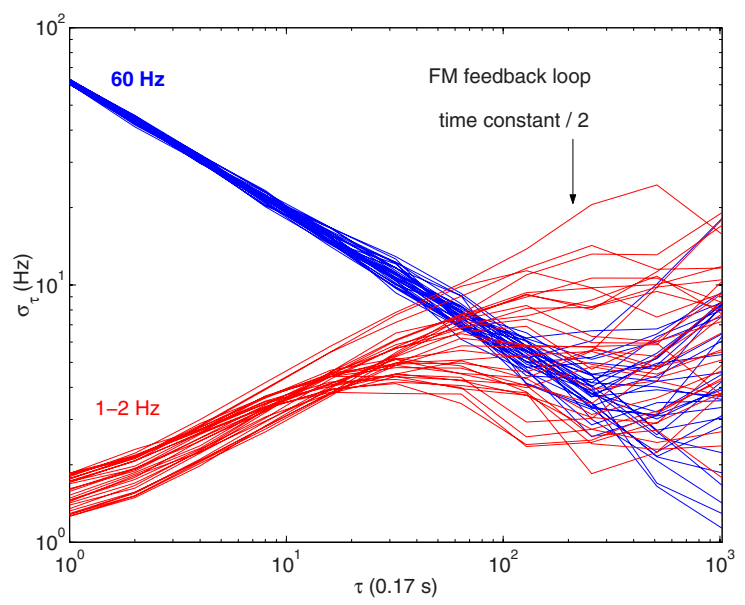

(b)

FIG. 3. (Color online) (a) Comparison of the standard deviation of the frequency measurement of a resonator located in a temperature-stabilized room and a 0.51 oil bath, as a function of FM excursion. These data were obtained using the embedded interrogation unit described in Ref. 1. The resonator is connected to the interrogation unit through a $26 \mathrm{~dB}$ attenuator, representative of typical rf propagation losses. Each experiment was performed multiple times and 3 representative data sets were exploited here. The top series of curves are the measurement for a fixed frequency comb (hence a result independent of FM excursion), and the bottom set of curves is obtained for the FM method. Each standard deviation is computed on 20 min time series, or 7200 points since the sampling rate is $6 \mathrm{~Hz}$. Different colors distinguish successive iterations of the same experiment. (b) The Allan deviation computed on the same data sets exhibits very different behaviors for the two interrogation strategies. The fixed comb method is intrinsically inaccurate due to the parabolic approximation of the resonance resulting from the magnitude measurements, and the accuracy improves with longer averaging times. On the other hand, the FM strategy is very accurate on the short term, but the time constant of the proportional feedback loop increases the noise level with longer integration time. Furthermore, this high short term accuracy magnifies the dependency with temperature fluctuations which could not be avoided with our crude stabilization setup. The colors encode the nature of the strategy, but all the curves acquired with different FM excursions were encoded with the same color since no significant difference was observed between the results.

range from -20 to $160{ }^{\circ} \mathrm{C}$, these figures translate as some $\mathrm{mK}$ temperature measurement resolution, which is to the best of our knowledge the best resolution ever obtained for wireless interrogation of surface acoustic wave sensors. ${ }^{4,5}$ The offset between the fixed comb method and FM strategies is discussed in Ref. 6.

The main drawbacks of this closed loop strategy is its reduced interrogation speed with respect to the fixed frequency comb method since the FM signal must be sampled during a few periods for phase identification: We have seen earlier that the modulation frequency is limited by the quality factor of the resonator. Hence, the interrogation speed (and hence measurement bandwidth) is reduced by a factor 10 . The strategy is nevertheless an invaluable improvement for high resolution, low bandwidth, wireless measurements.

Figure 3 shows the evolution of the standard deviation of the fixed frequency comb strategy and the FM strategy as a function of the FM excursion. The fixed frequency comb method exhibits a constant standard deviation of $65 \pm 5 \mathrm{~Hz}$ during the $30 \mathrm{~h}$ the experiment lasted. The FM strategy exhibits an optimum excursion around $10 \mathrm{kHz}$, which happens to be an optimum trade-off between immunity to magnitude measurement noise, while still staying below one third of the width at half height of the resonance [Fig. 3(a)]. This optimum value results in a noise level of about $1 \mathrm{~Hz}$, more than one order of magnitude improvement with respect to the fixed frequency comb method, yielding a relative stability of $2 \times 10^{-9}$ or a temperature resolution in the sub-mK range [Fig. 3(b)]. For comparison, the Hewlett Packard HP2830A oscillator used as reference in these measurements exhibits a noise level of $1 \mathrm{mHz}$ at $28 \mathrm{MHz}$, i.e., a relative stability of a few $10^{-11}$ at one second integration time, meaning the ability to measure $\mu \mathrm{K}$ temperature variations.
We have demonstrated a strategy for interrogating acoustic resonators based on the cancellation of an amplitude modulated signal resulting from the conversion of the FM emitted signal to an amplitude modulation by the transfer function of the resonator. A closed loop control of the emitted carrier frequency keeps the modulation frequency component of the returned signal minimum: This strategy results in a 10-fold improvement of the resonance frequency identification with respect to a fixed frequency-step magnitude envelope sampling and fit. This gain in resolution results however in a longer interrogation time since the modulation frequency must be slow with respect to the response time of the resonator, defined by its quality factor. Furthermore, the closed loop strategy of tracking the zero-phase condition makes the use of this strategy difficult when the sensor is only intermittently visible (e.g., rotating or moving object). Nevertheless, for a large number of applications, the system performance allows for reaching better resolutions than $\%$ (17 bit accuracy) over the whole measurement range.

${ }^{1}$ J.-M. Friedt, C. Droit, G. Martin, and S. Ballandras, Rev. Sci. Instrum. 81, 014701 (2010).

${ }^{2}$ B. K. Jones, Electronics for Experimentation and Research (Prentice Hall, Englewood Cliffs, 1986).

${ }^{3}$ S. Ballandras, G. Martin, and L. Fagot-Revurat, patent WO/2008/015129 available at http://www.wipo.int/pctdb/en/wo.jsp?wo $=2008015129$.

${ }^{4}$ W. E. Bulst, G. Fischerauer, and L. Reindl, IEEE Trans. Ind. Electron. 48, 265 (2001).

${ }^{5}$ L. M. Reindl and I. M. Shrena, IEEE Trans. Ultrason. Ferroelectr. Freq. Control 51, 1457 (2004).

${ }^{6}$ See supplementary material at http://dx.doi.org/10.1063/1.3402286 for a description of a tabletop experiment reproducing the results presented here with embedded hardware, and a complementary analysis of the influence of the FM excursion $B$ on the bias of the estimated resonance frequency. 